\title{
Multiple Positive Solutions for a Fractional Boundary Value Problem with Fractional Integral Deviating Argument
}

\author{
A. Guezane-Lakoud ${ }^{\mathbf{1}}$ and R. Khaldi ${ }^{2}$ \\ ${ }^{1}$ Laboratory of Advanced Materials, Faculty of Sciences, Badji Mokhtar-Annaba University, \\ P.O. Box 12, 23000 Annaba, Algeria \\ ${ }^{2}$ Laboratory LASEA, Faculty of Sciences, Badji Mokhtar-Annaba University, P.O. Box 12, \\ 23000 Annaba, Algeria
}

Correspondence should be addressed to R. Khaldi, rkhadi@yahoo.fr

Received 22 March 2012; Revised 23 May 2012; Accepted 29 May 2012

Academic Editor: Jean Michel Rakotoson

Copyright (C) 2012 A. Guezane-Lakoud and R. Khaldi. This is an open access article distributed under the Creative Commons Attribution License, which permits unrestricted use, distribution, and reproduction in any medium, provided the original work is properly cited.

This work is devoted to the existence of positive solutions for a fractional boundary value problem with fractional integral deviating argument. The proofs of the main results are based on GuoKrasnoselskii fixed point theorem and Avery and Peterson fixed point theorem. Two examples are given to illustrate the obtained results, ending the paper.

\section{Introduction}

The study of differential equations with deviating arguments has known a rapid development and still attracts most attention; it is a vast domain constantly enriched and fruitful. Differential equations with deviating arguments appear in many areas of sciences and technology such as in the study of problems related with combustion in rocket motion, in the theory of automatic control, in economics, and biological systems.

There exists a vast amount of literature devoted to the investigation of boundary value problems with deviating arguments (see [1-4]); one can cite the paper of Haloi et al. [5], where the authors studied an abstract initial value problem with deviating arguments by the help of Sobolevskii and Tanabe theory and fixed point theorems. However, few papers exist concerning fractional boundary value problems with deviating arguments, see $[3,4,6,7]$. We can cite the work of Jankowski [8], where the author studied the positivity of solutions for fourth-order differential equations with deviating arguments and integral boundary conditions, by Avery and Peterson theorem. 
Fractional differential equations have been proved to be valuable tools in the modelling of many phenomena in various fields of engineering, such as rheology, fluid flows, electrical networks, viscoelasticity, chemical physics, biosciences, signal processing, systems control theory, electrochemistry, mechanics and diffusion processes; for more details on this subject we refer the reader to [9-12].

In this work, we study a fractional boundary value problem with fractional deviating $\operatorname{argument}(\mathrm{P})$ :

$$
\begin{gathered}
{ }^{c} D_{0^{+}}^{q} u(t)+a(t) f\left(u\left(I_{0^{+}}^{\sigma} \theta(t)\right)\right)=0, \quad 0<t<1, \\
u^{\prime}(0)=u^{\prime \prime}(0)=0, \quad u(0)=\alpha u(1),
\end{gathered}
$$

where $2<q<3,0<\sigma<1,{ }^{c} D_{0^{+}}^{q}$ denotes the Caputo fractional derivative, $f \in C(\mathbb{R}, \mathbb{R})$ is a given function, $\theta \in C([0,1],[0,1]), a \in C([0,1], \mathbb{R})$. We assume that the deviating argument $\theta$ satisfies the delay property $0 \leq \theta(t) \leq t$ for any $t \in[0,1]$. Let us remark that when we apply the fractional integral $I_{0^{+}}^{\sigma}$ to the deviating argument $\theta$, then the problem $(\mathrm{P})$ becomes more delayed in time. The existence of a deviation delay in time is necessary in some situations, to avoid the unstable combustion in liquid rocket engine and to contribute in the conversion of the fuel mixture into the product of combustion. No contributions exist, as far as we know, concerning the positivity of solutions for the fractional differential equation with deviating argument $(\mathrm{P})$.

The literature on fractional boundary value problems with deviating argument is very reduced; we can cite Ntouyas et al. [6] work, where the authors investigated the existence of positive solutions to fractional differential equations with advanced arguments by GuoKrasnoselskii fixed point theorem. Other interesting results on fractional boundary value problems can be found in [13-21].

The organization of this paper is as follows. In Section 2, we provide some necessary background materials and definitions. In Section 3, we present some lemmas which are useful to obtain our main results. In Section 4, we discuss the existence of at least one positive solution of problem (P) by using Guo-Krasnoselskii fixed point theorem on cone; then, under some sufficient conditions on the nonlinear source term, we apply Avery-Peterson theorem to prove the existence of at least three positive solutions. At the end of this section, we give two examples to verify the obtained results.

\section{Background Materials and Definitions}

In this section, we introduce definitions from fractional calculus theory and preliminary facts which are used throughout this paper.

Definition 2.1 (see [10]). The integral

$$
I_{a^{+}}^{\alpha} g(t)=\frac{1}{\Gamma(\alpha)} \int_{a}^{t} \frac{g(s)}{(t-s)^{1-\alpha}} d s
$$

is called Riemann-Liouville fractional integral of $g$ of order $\alpha>0$ when the right side exists. Here $\Gamma$ is the gamma function. 
Definition 2.2 (see [10]). For a function $g$ given on the interval $[a, b]$, the Caputo fractional derivative of $g$ of order $\alpha$ is defined by

$$
{ }^{c} D_{a^{+}}^{\alpha} g(t)=\frac{1}{\Gamma(n-\alpha)} \int_{a}^{t} \frac{g^{(n)}(s)}{(t-s)^{\alpha-n+1}} d s,
$$

where $n=[\alpha]+1$ and $[\alpha]$ denotes the integer part of $\alpha$.

Lemma 2.3 (see [10]). Letting $\alpha, \beta>0$ and letting $n=[\alpha]+1$, then the following relations hold: ${ }^{c} D_{0^{+}}^{\alpha} t^{\beta-1}=(\Gamma(\beta) / \Gamma(\beta-\alpha)) t^{\beta-\alpha-1}, \beta>n$ and ${ }^{c} D_{0^{+}}^{\alpha} t^{k}=0, k=0,1,2, \ldots, n-1$.

Lemma 2.4 (see [10]). For $\alpha>0, g(t) \in C(0,1)$, the homogenous fractional differential equation

$$
{ }^{c} D_{a^{+}}^{\alpha} g(t)=0
$$

has a solution

$$
g(t)=c_{1}+c_{2} t+c_{3} t^{2}+\cdots+c_{n} t^{n-1}
$$

where $c_{i} \in R, i=0, \ldots, n$, and $n=[\alpha]+1$.

Denote by $L^{1}([0,1], \mathbb{R})$ the Banach space of Lebesgue integrable functions from $[0,1]$ into $\mathbb{R}$ with the norm $\|y\|_{L^{1}}=\int_{0}^{1}|y(t)| d t$. Define $E=C[0,1]$, equipped with the norm $\|u\|=$ $\max _{t \in[0,1]}|u(t)|$.

The following lemmas give some properties of Riemann-Liouville fractional integrals and Caputo fractional derivative.

Lemma 2.5 (see [10]). Let $p, q \geq 0, f \in L_{1}[a, b]$. Then $I_{0^{+}}^{p} I_{0^{+}}^{q} f(t)=I_{0^{+}}^{p+q} f(t)=I_{0^{+}}^{q} I_{0^{+}}^{p} f(t)$ and ${ }^{c} D_{0^{+}}^{q} I_{0^{+}}^{q} f(t)=f(t)$, for all $t \in[a, b]$.

Lemma 2.6 (see [10]). Let $\beta>\alpha>0$. Then the formula ${ }^{c} D_{0^{+}}^{\alpha} I_{0^{+}}^{\beta} f(t)=I_{0^{+}}^{\beta-\alpha} f(t)$ holds almost everywhere on $t \in[a, b]$, for $f \in L_{1}[a, b]$, and it is valid at any point $t \in[a, b]$ if $f \in C[a, b]$.

Now we present the necessary definitions from the theory of cone in Banach spaces.

Definition 2.7. A nonempty subset $P$ of a Banach space $E$ is called a cone if $P$ is convex, closed and satisfies the conditions

(i) $\alpha x \in P$ for all $x \in P$ and any $\alpha \in \mathbb{R}_{+}$,

(ii) $x,-x \in P$ imply $x=0$.

Definition 2.8. A map $F$ is said to be a nonnegative continuous convex functional on a cone $P$ of a real Banach space $E$ if $F: P \rightarrow[0, \infty$ [ is continuous and

$$
F(t x+(1-t) y) \leq t F(x)+(1-t) F(y), \quad \forall x, y \in P, t \in[0,1]
$$


Similarly $F$ is a nonnegative continuous concave functional on a cone $P$ of a real Banach space $E$ if

$$
F(t x+(1-t) y) \geq t F(x)+(1-t) F(y), \quad \forall x, y \in P, t \in[0,1]
$$

Definition 2.9. A mapping is called completely continuous if it is continuous and maps bounded sets into relatively compact sets.

In the following, we state some fixed point theorems.

Theorem 2.10 (Guo-Krasnoselskii fixed point theorem on cone [22]). Let $E$ be a Banach space, and let $K \subset E$ be a cone. Assume $\Omega_{1}$ and $\Omega_{2}$ are open subsets of $E$ with $0 \in \Omega_{1}, \overline{\Omega_{1}} \subset \Omega_{2}$ and let

$$
\mathcal{A}: K \cap\left(\overline{\Omega_{2}} \backslash \Omega_{1}\right) \rightarrow K
$$

be a completely continuous operator such that

(i) $\|\mathcal{A} u\| \leq\|u\|, u \in K \cap \partial \Omega_{1}$, and $\|\mathcal{A} u\| \geq\|u\|, u \in K \cap \partial \Omega_{2}$,

(ii) $\|\mathcal{A} u\| \geq\|u\|, u \in K \cap \partial \Omega_{1}$, and $\|\mathcal{A} u\| \leq\|u\|, u \in K \cap \partial \Omega_{2}$.

Then $A$ has a fixed point in $K \cap\left(\overline{\Omega_{2}} \backslash \Omega_{1}\right)$.

Theorem 2.11 (Avery and Peterson fixed point theorem [23]). Let $P$ be a cone in a real Banach space $E$. Let $\varphi$ and $\Phi$ be continuous, nonnegative, and convex functionals on $P$, let $\Lambda$ be a continuous nonnegative, and concave functional on $P$, and let $\Psi$ be continuous and nonnegative functional on $P$ satisfying $\Psi(k u) \leq k\|u\|$ for $0 \leq k \leq 1$. Define the sets, $P(\varphi, d)=\{u \in P, \varphi(u)<d\}, P(\varphi, \Lambda, b, d)=$ $\{u \in P, b \leq \Lambda(u), \varphi(u) \leq d\}, P(\varphi, \Phi, \Lambda, b, c, d)=\{u \in P, b \leq \Lambda(u), \Phi(u) \leq c, \varphi(u) \leq d\}$, $R(\varphi, \Psi, a, d)=\{u \in P, a \leq \Psi(u), \varphi(u) \leq d\}$. For $M$ and $d$ positive numbers we have $\Lambda(u) \leq \Psi(u)$ and $\|u\| \leq M \varphi(u)$ for any $u \in \overline{P(\varphi, d)}$. Assume $T: \overline{P(\varphi, d)} \rightarrow \overline{P(\varphi, d)}$ is completely continuous and there exists positive numbers $a, b$, and $c$ with $a<b$ such that

(S1) $\{u \in P(\varphi, \Phi, \Lambda, b, c, d), \Lambda(u)>b\} \neq \emptyset$ and $\Lambda(T u)>b$ for $u \in P(\varphi, \Phi, \Lambda, b, c, d)$,

(S2) $\Lambda(T u)>b$ for $u \in P(\varphi, \Lambda, b, d)$ with $\Phi(T u)>c$,

(S3) $0 \notin R(\varphi, \Psi, a, d)$ and $\Psi(T u)<a$ for $u \in R(\varphi, \Psi, a, d)$ with $\Psi(u)=a$.

Then $T$ has at least three positive fixed points $u_{1}, u_{2}, u_{3} \in \overline{P(\varphi, d)}$ such that $\varphi\left(u_{i}\right) \leq d$, for $i=1,2,3 . b<\Lambda\left(u_{1}\right), a<\Psi\left(u_{2}\right)$, with $\Lambda\left(u_{2}\right)<b$ and $\Psi\left(u_{3}\right)<a$.

\section{Some Lemmas}

We start by solving an auxiliary problem which allows us to get the expression of the solution. Let us consider the following problem:

$$
\begin{gathered}
{ }^{c} D_{0^{+}}^{q} u(t)+y(t)=0, \quad 0<t<1, \\
u^{\prime}(0)=u^{\prime \prime}(0)=0, \quad u(0)=\alpha u(1) .
\end{gathered}
$$


Lemma 3.1. Assuming that $\alpha \neq 1$ and $y \in C([0,1], \mathbb{R})$, then the problem $(P 1)$ has a unique solution given by

$$
u(t)=\frac{1}{\Gamma(q)} \int_{0}^{1} G(t, s) y(s) d s
$$

where

$$
G(t, s)= \begin{cases}-(t-s)^{q-1}+\frac{\alpha}{\alpha-1}(1-s)^{q-1}, & 0 \leq s \leq t \\ \frac{\alpha}{\alpha-1}(1-s)^{q-1}, & t \leq s \leq 1\end{cases}
$$

Proof. Using Lemmas 2.3 and 2.4, we get

$$
u(t)=-I_{0^{+}}^{q} y(t)+a+b t+c t^{2} .
$$

The boundary conditions $u^{\prime}(0)=u^{\prime \prime}(0)=0$ give $b=c=0$, and the condition $u(0)=\alpha u(1)$ implies $a=(\alpha /(\alpha-1)) I_{0^{+}}^{q} y(1)$. Substituting $a, b$, and $c$ by their values in (3.3) we get the desired result.

Some useful estimates of the Green function $G$ are given hereafter.

Lemma 3.2. If $\alpha>1$, then for all $s, t \in[0,1]$, the Green function $G(t, s)$ is nonnegative, continuous and satisfies

(i) $\max _{s, t \in[0,1]} G(t, s)=\alpha /(\alpha-1)$,

(ii) $\min _{s, t \in[0, \tau]} G(t, s)=(1-\tau)^{q-1} /(\alpha-1)$ where $0<\tau<1$.

Proof. The proof is easy; then we omit it.

Lemma 3.3. Assuming that $\alpha>1$ and $y \in C\left([0,1], \mathbb{R}_{+}\right)$, then the unique solution of problem (P1) satisfies $u(t) \geq 0$ on $[0,1]$. Moreover we have

$$
\min _{t \in[0, \tau]} u(t) \geq \frac{1-\tau^{2}}{\alpha}\|u\|
$$

Proof. From $u^{\prime \prime}(t)=-I_{0^{+}}^{q-2} y(t)$ and hypothesis on the function $y$ we deduce that $u^{\prime \prime} \leq 0$ consequently the function $u$ is concave. Moreover from the condition $u(0)=\alpha u(1)$ we can say that the values $u(0)$ and $u(1)$ have the same sign. Since $u(0)=(\alpha /(\alpha-1)) I_{0^{+}}^{q} y(1) \geq 0$, then $u(t) \geq 0$ on $[0,1]$. Let us analyze the boundary conditions; from $u^{\prime \prime} \leq 0$ and the condition $u^{\prime}(0)=0$ we conclude that $u^{\prime}$ is also negative; consequently the function $u$ is decreasing. From the above discussion we conclude that $\max _{t \in[0,1]} u(t)=u(0)$ and $\min _{t \in[0, \tau]} u(t)=u(\tau)$. The concavity of $u$ allows to write

$$
\frac{u(\tau)-u(0)}{\tau} \geq \frac{u(1)-u(\tau)}{1-\tau}
$$


Using the condition $u(0)=\alpha u(1)$ it yields

$$
u(\tau)-u(0) \geq \frac{u(0)}{\alpha} \frac{\tau}{1-\tau}-u(\tau) \frac{\tau}{(1-\tau)},
$$

then

$$
u(\tau)\left(1+\frac{\tau}{1-\tau}\right) \geq u(0)\left(1+\frac{\tau}{\alpha(1-\tau)}\right)
$$

Since $(1 /(1-\tau))>1$, we have

$$
u(\tau) \frac{1}{1-\tau} \geq u(0)\left(1+\frac{\tau}{\alpha(1-\tau)}\right) \geq u(0)\left(1+\frac{\tau}{\alpha}\right)
$$

so,

$$
u(\tau) \geq u(0)(1-\tau)\left(\frac{\alpha+\tau}{\alpha}\right) .
$$

Using the assumption $\alpha>1$, we obtain

$$
u(\tau) \geq \frac{1-\tau^{2}}{\alpha} u(0)
$$

consequently

$$
\min _{t \in[0, \tau]} u(t) \geq \frac{1-\tau^{2}}{\alpha}\|u\|
$$

This achieves the proof.

\section{Existence of Positive Solutions}

We first state the assumptions that will be used to prove our main results:

(H1) $\theta \in C([0,1],[0,1])$ and satisfies the delay property $0 \leq \theta(t) \leq t$, for all $t \in[0,1]$,

(H2) $a \in C\left([0,1], \mathbb{R}_{+}\right)$and $\int_{0}^{\tau} a(s) d s \neq 0$,

(H3) $f \in C\left(\mathbb{R}_{+}, \mathbb{R}_{+}\right)$and $\alpha>1$.

Define the operator $T: E \rightarrow E$ by

$$
T u(t)=\frac{1}{\Gamma(q)} \int_{0}^{1} G(t, s) a(s) f\left(u\left(I_{0^{+}}^{\sigma} \theta(s)\right)\right) d s .
$$


Definition 4.1. A function $u$ is called positive solution of problem (P) if $u(t) \geq 0$, for all $t \in$ $[0,1]$ and it satisfies the boundary conditions in $(\mathrm{P})$.

Let us introduce the following notations $A_{0}=\lim _{u \rightarrow 0^{+}}(f(u) / u), A_{\infty}=$ $\lim _{u \rightarrow \infty}(f(u) / u)$. The case $A_{0}=0$ and $A_{\infty}=\infty$ is called superlinear case, and the case $A_{0}=\infty$ and $A_{\infty}=0$ is called sublinear case.

Theorem 4.2. Assuming that (H1)-(H3) hold, then the problem (P) has at least one positive solution in both cases, superlinear as well as sublinear.

Proof. We apply Guo-Krasnoselskii fixed point theorem on cone. Define the cone $P=\{u \in$ $E$, $u$ non negative on $[0,1]$ and $\left.\min _{t \in[0, \tau]} u(t) \geq\left(\left(1-\tau^{2}\right) / \alpha\right)\|u\|\right\}$. It is easy to check that $P$ is a nonempty closed and convex subset of $E$, so it is a cone. One can check that $T(P) \subset P$. It is obvious that $T$ is continuous since $G, a$ and $f$ are continuous. Let us prove that $T$ is completely continuous mapping on $P$.

Claim 1. $T\left(B_{r}\right)$ is uniformly bounded, where $B_{r}=\{u \in P,\|u\| \leq r\}$. Let us remark that if $u \in P$, then

$$
\min _{t \in[0, \tau]} u\left(I_{0^{+}}^{\sigma} \theta(t)\right) \geq \frac{1-\tau^{2}}{\alpha}\|u\|
$$

In fact from the delay property, the properties of Riemann fractional integrals and $0<\sigma<1$, we have

$$
0 \leq I_{0^{+}}^{\sigma} \theta(t) \leq \frac{t^{\sigma+1}}{\Gamma(\sigma+2)} \leq 1
$$

In view of the concavity of $u$ it yields

$$
u\left(I_{0^{+}}^{\sigma} \theta(t)\right) \geq \min _{t \in[0, \tau]} u(t) \geq \frac{1-\tau^{2}}{\alpha}\|u\|
$$

Therefore

$$
\min _{t \in[0, \tau]} u\left(I_{0^{+}}^{\sigma} \theta(t)\right) \geq \min _{t \in[0, \tau]} u(t) \geq \frac{1-\tau^{2}}{\alpha}\|u\| \geq \frac{1-\tau^{2}}{\alpha}\left\|u\left(I_{0^{+}}^{\sigma} \theta\right)\right\|
$$

Since the functions $a$ and $f$ are continuous, then there exists a constant $k$ such that $\max _{t \in[0,1]}\left|a(t) f\left(u\left(I_{0^{+}}^{\sigma} \theta(t)\right)\right)\right|=k$ for any $u \in B_{r}$. By virtue of Lemma 3.2 we obtain

$$
|T u(t)| \leq \frac{k \alpha}{(\alpha-1) \Gamma(q)} .
$$

Hence $T$ is uniformly bounded. 
Claim 2. $T$ is equicontinuous. We have for any $u \in B_{r}$

$$
\begin{aligned}
\left|T^{\prime} u(t)\right| & =\left|\frac{q-1}{\Gamma(q)} \int_{0}^{t}(t-s)^{q-2} a(s) f\left(u(s), u\left(I^{\sigma} \theta(s)\right)\right) d s\right| \\
& \leq \frac{k}{\Gamma(q-1)} \int_{0}^{1}(1-s)^{q-2} d s=\frac{k}{\Gamma(q)} .
\end{aligned}
$$

Therefore

$$
\left|T u\left(t_{2}\right)-T u\left(t_{1}\right)\right|=\left|\int_{t_{1}}^{t_{2}} T^{\prime} u(t) d t\right| \leq \frac{k\left(t_{2}-t_{1}\right)}{\Gamma(q)} .
$$

Thus $T$ is equicontinuous; from Ascoli-Arzela Theorem, we deduce that $T$ is completely continuous.

Now, we prove the sublinear case. Since $A_{0}=\infty$, then for any $A>0$ there exists $\delta>0$, such that for any $u, 0<u \leq \delta$ then $f(u) \geq$ A $u$. Set $\Omega_{1}=\{u \in E:\|u\|<\delta\}$. Letting $u \in P \cap \partial \Omega_{1}$, we can see that $u\left(I_{0^{+}}^{\sigma} \theta\right) \in P \cap \partial \Omega_{1}$; then from (4.5) we have

$$
\begin{aligned}
\|T u\| & =\max _{t \in[0,1]}|T u(t)|=\frac{\alpha}{(\alpha-1) \Gamma(q)} \int_{0}^{1} a(s) f\left(u\left(I_{0^{+}}^{\sigma} \theta(s)\right)\right) d s \\
& \geq \frac{A \alpha}{(\alpha-1) \Gamma(q)} \int_{0}^{1} a(s) u\left(I_{0^{+}}^{\sigma} \theta(s)\right) d s .
\end{aligned}
$$

With the help of (4.4) we get

$$
\|T u\| \geq \frac{A\left(1-\tau^{2}\right)\|u\|}{\Gamma(q)} \int_{0}^{1} a(s) d s .
$$

In view of assumption (H2) we can choose $A=\Gamma(q) /\left(1-\tau^{2}\right) \int_{0}^{1} a(s) d s$; therefore (4.10) becomes $\|T u\| \geq\|u\|$.

On the other hand since $A_{\infty}=0$, we deduce that for any $\varepsilon>0$ there exists $\gamma>0$, such that for any $u, u \geq \gamma$ then $f(u) \leq \varepsilon u$. Setting $R=\max \{2 \delta,((\alpha-1) / \alpha) \gamma\}$ and $\Omega_{2}=\{u \in E:\|u\|<$ $R\}$, then $\overline{\Omega_{1}} \subset \Omega_{2}$ and for $u \in P \cap \partial \Omega_{2}$ we have $\min _{t \in[0, \tau]} u(t) \geq(\alpha /(\alpha-1))\|u\|=\alpha R /(\alpha-1) \geq \gamma$; consequently $u\left(I_{0^{+}}^{\sigma} \theta(t)\right) \geq \gamma$. Taking Lemma 3.2 and (4.5) into account it yields

$$
\begin{aligned}
T u(t) & \leq \frac{\alpha}{(\alpha-1) \Gamma(q)} \int_{0}^{1} a(s) f\left(u\left(I_{0^{+}}^{\sigma} \theta(s)\right)\right) d s \\
& \leq \frac{\alpha \varepsilon}{(\alpha-1) \Gamma(q)} \int_{0}^{1} a(s) u\left(I_{0^{+}}^{\sigma} \theta(s)\right) d s \\
& \leq \frac{\alpha \varepsilon\left\|u\left(I_{0^{+}}^{\sigma} \theta\right)\right\|}{(\alpha-1) \Gamma(q)} \int_{0}^{1} a(s) d s \leq \frac{\alpha \varepsilon\|u\|}{(\alpha-1) \Gamma(q)} \int_{0}^{1} a(s) d s .
\end{aligned}
$$


Then if we choose $\varepsilon=(\alpha-1) \Gamma(q) / \alpha \int_{0}^{1} a(s) d s$, we get $\|T u\| \leq\|u\|$ for all $u \in P \cap \partial \Omega_{2}$. The second part of Theorem 2.10 implies that $T$ has a fixed point in $P \cap\left(\overline{\Omega_{2}} \backslash \Omega_{1}\right)$; that means that $T$ has at least a positive solution in $P \cap\left(\overline{\Omega_{2}} \backslash \Omega_{1}\right)$. Arguing as above, we prove the superlinear case. The proof is complete.

Let us introduce the following functionals. Defining on $P$, the nonnegative, continuous, and concave functional $\Lambda$ by $\Lambda(u)=\min _{t \in[0, \tau]}|u(t)|$, then $\Lambda(u) \leq\|u\|$. Defining the nonnegative, continuous, and convex functionals $\varphi$ and $\Phi$ on $P$ by $\varphi(u)=\Phi(u)=\|u\|$ and the nonnegative continuous functional $\Psi$ on $P$ by $\Psi(u)=\|u\|$, then $\Psi(k u) \leq k\|u\|$ for $0 \leq k \leq 1$.

Theorem 4.3. Let assumptions (H1)-(H3) hold, and assume that there exist positive constants $a, b, c, d, \mu, L$ such that $a<b, \mu>(\alpha /(\alpha-1) \Gamma(q)) \int_{0}^{1} a(s) d s, L<\left(\alpha(1-\tau)^{q-1} /(\alpha-\right.$ 1) $\Gamma(q)) \int_{0}^{\tau} a(s) d s$ and

(i) $f(u) \leq d / \mu$ for $u \in[0, d]$,

(ii) $f(u) \geq b / L$ for $u \in[b, b / \xi]$,

(iii) $f(u) \leq a / \mu$ for $u \in[0, a]$.

Then problem $(P)$ has at least three positive solutions $u_{1}, u_{2}, u_{3} \in \overline{P(\varphi, b)}$ such that $\varphi\left(u_{i}\right) \leq d$, for $i=1,2,3 . b<\Lambda\left(u_{1}\right), a<\Psi\left(u_{2}\right)$, with $\Lambda\left(u_{2}\right)<b$ and $\Psi\left(u_{3}\right)<a$.

Proof. To prove the existence of three positive solutions, we apply Theorem 2.11. Proceeding analogously as in the proof of Theorem 4.2, we prove that the mapping $T$ is completely continuous on $\overline{P(\varphi, d)}$.

Claim 1. T( $\overline{P(\varphi, d)}) \subset \overline{P(\varphi, d)}$.

Letting $u \in \overline{P(\varphi, d)}$, then $\|u\| \leq d$; since $0<\theta(t)<t$, for all $t \in[0,1]$, then in view of (4.5) we have $u\left(I_{0^{+}}^{\sigma} \theta(t)\right) \leq\left\|u\left(I_{0^{+}}^{\sigma} \theta\right)\right\| \leq\|u\| \leq d$. Thus with the help of assumption (i) it yields

$$
\begin{aligned}
\varphi(T u) & =\|T u\|=\max _{t \in[0,1]} \frac{1}{\Gamma(q)} \int_{0}^{1} G(t, s) a(s) f\left(u\left(I_{0^{+}}^{\sigma} \theta(s)\right)\right) d s \\
& \leq \frac{\alpha}{(\alpha-1) \Gamma(q)} \int_{0}^{1} a(s) f\left(u\left(I_{0^{+}}^{\sigma} \theta(s)\right)\right) d s \\
& \leq \frac{d}{\mu} \frac{\alpha}{(\alpha-1) \Gamma(q)} \int_{0}^{1} a(s) d s<d
\end{aligned}
$$

and hence $T u \in \overline{P(\varphi, d)}$.

Claim 2. (S1) holds; that is, $\{u \in P(\varphi, \Phi, \Lambda, b, b / \xi, d), \Lambda(u)>b\} \neq \emptyset$ and $\Lambda(T u)>b$ for $u \in P(\varphi, \Phi, \Lambda, b, b / \xi, d)$. Let $x(t)=(b / 2)(1+1 / \xi)$; since $\alpha>1$ and $\xi<1$, then

$$
\Phi(x)=\varphi(x)=\|x\|=\frac{b}{2}\left(1+\frac{1}{\xi}\right)=\frac{b}{2 \xi}(\xi+1)<\frac{b}{\xi} .
$$


Moreover we have

$$
\Lambda(x)=\min _{t \in[0, \tau]} x(t)=\frac{b}{2}\left(1+\frac{1}{\xi}\right)>b>\xi\|x\| .
$$

Thus $x \in P(\varphi, \Phi, \Lambda, b, b / \xi, d)$, so $\{u \in P(\varphi, \Phi, \Lambda, b, b / \xi, d), \Lambda(u)>b\} \neq \emptyset$.

Letting $u \in P(\varphi, \Phi, \Lambda, b, b / \xi, d)$, then $b \leq u(t) \leq b / \xi$ and $b \leq u\left(I_{0^{+}}^{\sigma} \theta(t)\right) \leq b / \xi$; thus by virtue of Lemma 3.2 and assumption (ii), we obtain

$$
\begin{aligned}
\Lambda(T u) & =\min _{t \in[0, \tau]}|T u(t)| \geq \frac{(1-\tau)^{q-1}}{(\alpha-1) \Gamma(q)} \int_{0}^{\tau} a(s) f\left(u\left(I_{0^{+}}^{\sigma} \theta(s)\right)\right) d s \\
& \geq \frac{(1-\tau)^{q-1} b}{(\alpha-1) \Gamma(q) L} \int_{0}^{\tau} a(s) d s>b .
\end{aligned}
$$

So condition (S1) is satisfied.

Claim 3. (S2) holds. Letting $u \in P(\varphi, \Lambda, b, d)$ such $\Phi(T u)=\|T u\|>b / \xi$, then

$$
\Lambda(T u)=\min _{t \in[0, \tau]}|T u(t)| \geq \xi\|T u\|>b
$$

This implies that claim (S2) holds true.

Claim 4. (S3) holds. Letting $u \in R(\varphi, \Psi, a, d)$, then $0<a \leq\|u\| \leq d$, and then $0 \notin$ $R(\varphi, \Psi, a, d)$. Let $u \in R(\varphi, \Psi, a, d)$ with $\Psi(u)=\|u\|=a$; using Lemma 3.2 and assumption (iii) it yields

$$
\begin{aligned}
\Psi(T u) & =\max _{t \in[0,1]} \frac{1}{\Gamma(q)} \int_{0}^{1} G(t, s) a(s) f\left(u\left(I_{0^{+}}^{\sigma} \theta(s)\right)\right) d s \\
& =\frac{\alpha}{(\alpha-1) \Gamma(q)} \int_{0}^{1} a(s) f\left(u\left(I_{0^{+}}^{\sigma} \theta(s)\right)\right) d s \\
& \leq \frac{a}{\mu} \frac{\alpha}{(\alpha-1) \Gamma(q)} \int_{0}^{1} a(s) d s<a .
\end{aligned}
$$

Then (S3) is satisfied.

Finally we conclude by Theorem 2.11 that there exist at least three positive solutions $u_{1}, u_{2}, u_{3} \in \overline{P(\varphi, d)}$ such that $\varphi\left(u_{i}\right) \leq d$, for $i=1,2,3 . b<\Lambda\left(u_{1}\right), a<\Psi\left(u_{2}\right)$, with $\Lambda\left(u_{2}\right)<b$ and $\Psi\left(u_{3}\right)<a$. The proof of Theorem 4.2 is complete.

Remark 4.4. Contrary to paper [8], we cannot consider problem $(\mathrm{P})$ with $0 \leq t \leq \theta(t) \leq 1$; in fact $I_{0^{+}}^{\sigma} \theta(t)$ can be greater than 1 for $0<\sigma<1$.

Now we give two examples to illustrate Theorems 4.2 and 4.3. 
Example 4.5. Let us consider the following fractional boundary value problem with fractional deviating argument:

$$
\begin{gathered}
{ }^{c} D_{0^{+}}^{7 / 3} u(t)+a(t) f\left(u\left(I_{0^{+}}^{\sigma} \theta(t)\right)\right)=0, \quad 0<t<1, \\
u^{\prime}(0)=u^{\prime \prime}(0)=0, \quad u(0)=\frac{5}{2} u(1),
\end{gathered}
$$

where $q=7 / 3, \sigma=3 / 5, \alpha=5 / 2, f(u)=\exp (-u), \theta(t)=t(1-t) \leq t, a(t)=\sqrt{1+t}, \tau=9 / 10$. By simple calculus we obtain $I_{0^{+}}^{3 / 5} \theta(t)=(1 / \Gamma(3 / 5))(t)^{3 / 5}\left((25 / 24) t-(125 / 156) t^{2}\right), \int_{0}^{9 / 10} a(s) d s=$ 1. $0793 \neq 0$. It is easy to check that the assumptions (H1)-(H3) hold and that $A_{0}=\infty, A_{\infty}=0$. Applying Theorem 4.2 we deduce that there exists at least one positive solution.

Example 4.6. Let us consider the fractional boundary value problem with fractional deviating argument:

$$
\begin{gathered}
{ }^{c} D_{0^{+}}^{5 / 2} u(t)+a(t) f\left(u\left(I_{0^{+}}^{\sigma} \theta(t)\right)\right)=0, \quad 0<t<1, \\
u^{\prime}(0)=u^{\prime \prime}(0)=0, \quad u(0)=2 u(1),
\end{gathered}
$$

where

$$
\begin{aligned}
& q=\frac{5}{2}, \quad \sigma=\frac{1}{2}, \quad \alpha=2, \quad \theta(t)=t^{2} \leq t, \quad a(t)=1+t, \\
& f(u)= \begin{cases}\frac{u^{2}}{5}, & 0 \leq u \leq 2 \\
\frac{301}{5} u-\frac{598}{5}, & 2 \leq u \leq 3 \\
61, & u \geq 3 .\end{cases}
\end{aligned}
$$

It is easy to see that $I_{0^{+}}^{1 / 2} \theta(t)=(16 / 15 \pi) t^{5 / 2} \leq 1$ and that hypotheses $(\mathrm{H} 1)-(\mathrm{H} 3)$ are satisfied. Let us check the assumptions of Theorem 4.2 for $\tau=4 / 5$ :

$$
\begin{gathered}
\mu>\frac{2}{\Gamma(5 / 2)} \int_{0}^{1}(1+s) d s=\frac{4}{\sqrt{\pi}}=2.2568 \\
L<\frac{\alpha(1-\tau)^{q-1}}{(\alpha-1) \Gamma(q)} \int_{0}^{\tau} a(s) d s=0.15072
\end{gathered}
$$

If we choose $\mu=2.5, L=0.05, a=2, b=3, d \geq 305 / 2, \xi=50 / 3, c=9 / 50$, then the assumptions of Theorem 4.2 are satisfied; consequently, there exist at least three positive solutions $u_{1}, u_{2}, u_{3} \in \overline{P(\varphi, d)}$ such that $\left\|u_{i}\right\| \leq d=153,3<\min _{t \in[0,4 / 5]} u_{1}(t), 2<\left\|u_{2}\right\|$, with $\min _{t \in[0,4 / 5]} u_{2}(t)<3$ and $\left\|u_{3}\right\|<2$. 


\section{Acknowledgment}

The authors would like to express their thanks to the referee for his helpful comments and suggestions.

\section{References}

[1] T. Jankowski, "Positive solutions for three-point one-dimensional $p$-Laplacian boundary value problems with advanced arguments," Applied Mathematics and Computation, vol. 215, no. 1, pp. 125$131,2009$.

[2] T. Jankowski, "Solvability of three point boundary value problems for second order differential equations with deviating arguments," Journal of Mathematical Analysis and Applications, vol. 312, no. 2, pp. 620-636, 2005.

[3] J. Yan, "Oscillation of first-order impulsive differential equations with advanced argument," Computers \& Mathematics with Applications, vol. 42, no. 10-11, pp. 1353-1363, 2001.

[4] Ch. Yang, Ch. Zhai, and J. Yan, "Positive solutions of the three-point boundary value problem for second order differential equations with an advanced argument," Nonlinear Analysis, vol. 65, no. 10, pp. 2013-2023, 2006.

[5] R. Haloi, D. Bahuguna, and D. N. Pandey, "Existence and uniqueness of solutions for quasi-linear differential equations with deviating arguments," Electronic Journal of Differential Equations, vol. 2012, no. 13, pp. 1-10, 2012.

[6] S. K. Ntouyas, G. Wang, and L. Zhang, "Positive solutions of arbitrary order nonlinear fractional differential equations with advanced arguments," Opuscula Mathematica, vol. 31, no. 3, pp. 433-442, 2011.

[7] G. Wang, S. K. Ntouyas, and L. Zhang, "Positive solutions of the three-point boundary value problem for fractional-order differential equations with an advanced argument," Advances in Difference Equations, vol. 2011, article 2, 2011.

[8] T. Jankowski, "Positive solutions for fourth-order differential equations with deviating arguments and integral boundary conditions," Nonlinear Analysis, vol. 73, no. 5, pp. 1289-1299, 2010.

[9] B. Bonilla, M. Rivero, L. Rodríguez-Germá, and J. J. Trujillo, "Fractional differential equations as alternative models to nonlinear differential equations," Applied Mathematics and Computation, vol. 187, no. 1, pp. 79-88, 2007.

[10] A. A. Kilbas, H. M. Srivastava, and J. J. Trujillo, Theory and Applications of Fractional Differential Equations, vol. 204 of North-Holland Mathematics Studies, Elsevier, Amsterdam, The Netherlands, 2006.

[11] V. Lakshmikantham and A. S. Vatsala, "Basic theory of fractional differential equations," Nonlinear Analysis, vol. 69, no. 8, pp. 2677-2682, 2008.

[12] I. Podlubny, Fractional Differential Equations, vol. 198 of Mathematics in Science and Engineering, Academic Press, San Diego, Calif, USA, 1999.

[13] B. Ahmad and J. J. Nieto, "Existence results for a coupled system of nonlinear fractional differential equations with three-point boundary conditions," Computers $\mathcal{E}$ Mathematics with Applications, vol. 58, no. 9, pp. 1838-1843, 2009.

[14] B. Ahmad and J. J. Nieto, "Anti-periodic fractional boundary value problems," Computers $\mathcal{E}$ Mathematics with Applications, vol. 62, no. 3, pp. 1150-1156, 2011.

[15] B. Ahmad, J. J. Nieto, and J. Pimentel, "Some boundary value problems of fractional differential equations and inclusions," Computers \& Mathematics with Applications, vol. 62, no. 3, pp. 1238-1250, 2011.

[16] Z. Bai, "On positive solutions of a nonlocal fractional boundary value problem," Nonlinear Analysis, vol. 72, no. 2, pp. 916-924, 2010.

[17] M. Benchohra, S. Hamani, and S. K. Ntouyas, "Boundary value problems for differential equations with fractional order and nonlocal conditions," Nonlinear Analysis, vol. 71, no. 7-8, pp. 2391-2396, 2009.

[18] A. Guezane-Lakoud and R. Khaldi, "Solvability of a fractional boundary value problem with fractional integral condition," Nonlinear Analysis, vol. 75, no. 4, pp. 2692-2700, 2012.

[19] A. Guezane-Lakoud and R. Khaldi, "Solvability of a two-point fractional bound-ary value problem," The Journal of Nonlinear Science and Applications, vol. 5, pp. 64-73, 2012. 
[20] A. Guezane-Lakoud and R. Khaldi, "Positive solution to a higher order fractional boundary value problem with fractional integral condition," Romanian Journal of Mathematics and Computer Sciences, vol. 2, pp. 28-40, 2012.

[21] G. Wang, B. Ahmad, and L. Zhang, "Impulsive anti-periodic boundary value problem for nonlinear differential equations of fractional order," Nonlinear Analysis, vol. 74, no. 3, pp. 792-804, 2011.

[22] D. J. Guo and V. Lakshmikantham, Nonlinear Problems in Abstract Cones, vol. 5 of Notes and Reports in Mathematics in Science and Engineering, Academic Press, San Diego, Calif, USA, 1988.

[23] R. I. Avery and A. C. Peterson, "Three positive fixed points of nonlinear operators on ordered Banach spaces," Computers \& Mathematics with Applications, vol. 42, no. 3-5, pp. 313-322, 2001. 


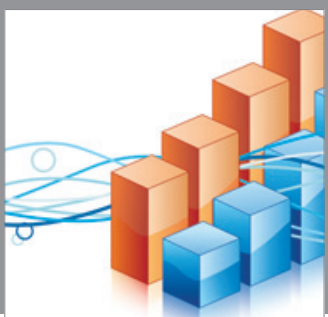

Advances in

Operations Research

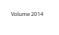

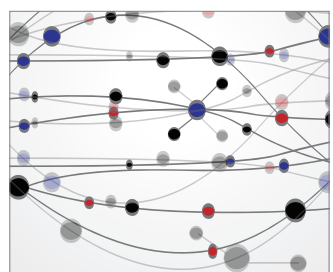

\section{The Scientific} World Journal
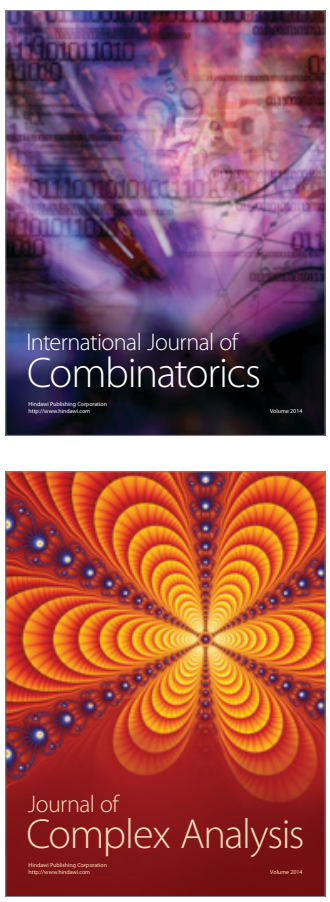

International Journal of

Mathematics and

Mathematical

Sciences
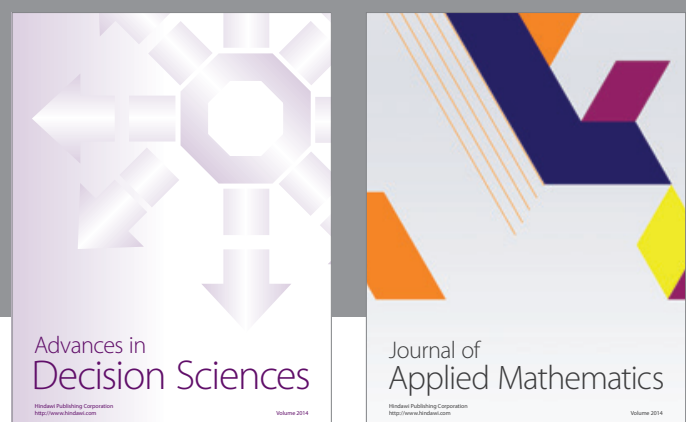

Journal of

Applied Mathematics
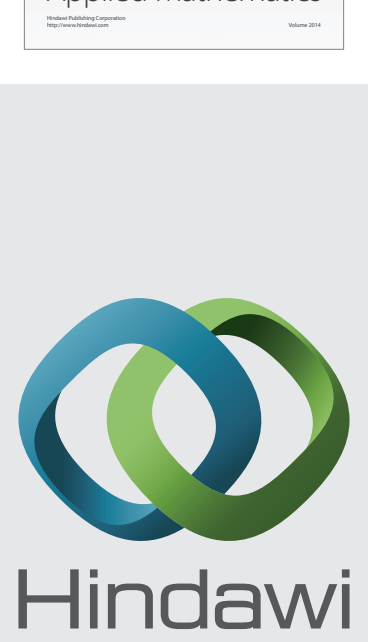

Submit your manuscripts at http://www.hindawi.com
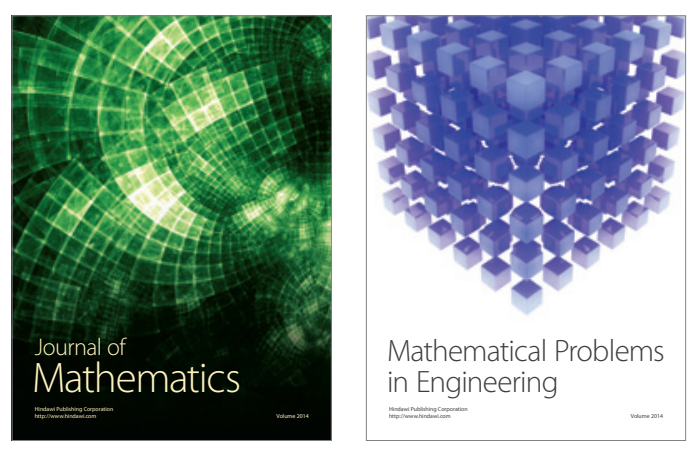

Mathematical Problems in Engineering
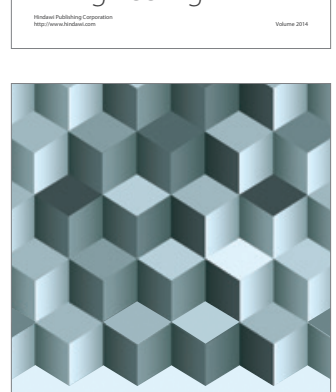

Journal of

Function Spaces
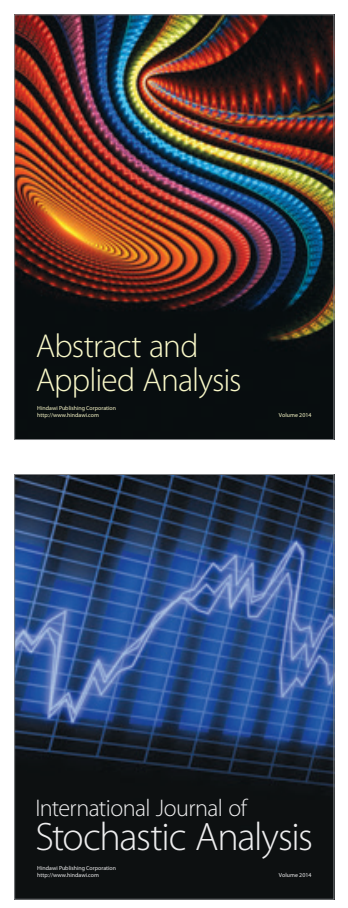

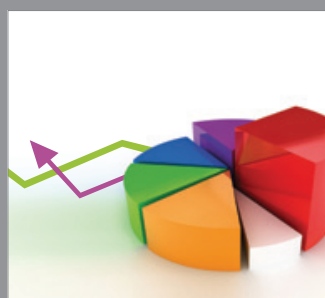

ournal of

Probability and Statistics

Promensencen
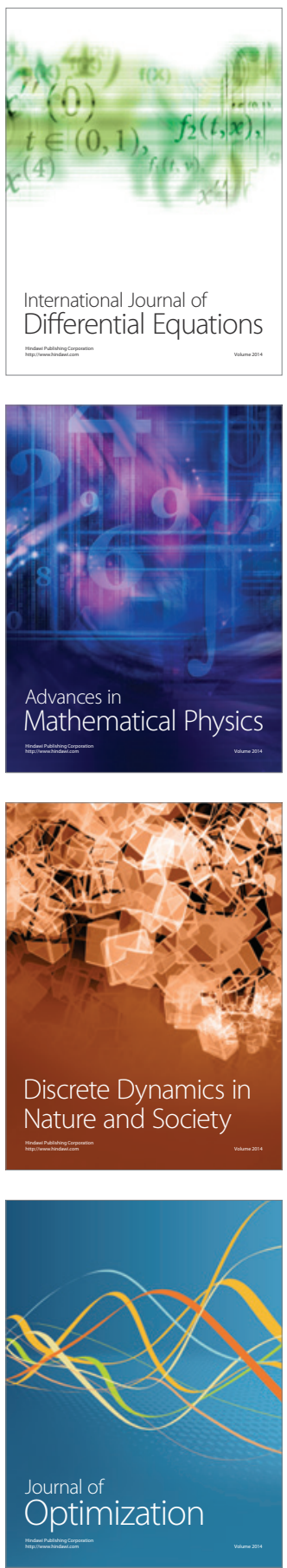